\title{
Long-term clinical outcomes of Toric IOL and ART +3.0D IOL in patients with cataract and corneal astigmatism
}

\author{
jie luo \\ Harbin Medical University https://orcid.org/0000-0001-9497-9480 \\ Yang Liu \\ Daqing Oilfield General Hospital \\ Feng Wang ( $\sim$ doctorhrb0451@163.com ) \\ Ying Su \\ Harbin Medical University \\ Xuebing Xiao \\ Daqing Oilfield General Hospital
}

Research article

Keywords: Cataract, Astigmatism, Intraocular lens, Long-term

Posted Date: October 29th, 2019

DOI: https://doi.org/10.21203/rs.2.16560/v1

License: (c) (1) This work is licensed under a Creative Commons Attribution 4.0 International License. Read Full License 


\section{Abstract}

Background We aimed to evaluate the long-term clinical outcomes of Toric intraocular lens (Toric IOL)and Acrysof IQ Restor Toric +3.0D intraocular lens (ART +3.0D IOL)in patients with cataract and corneal astigmatism.

Methods Fifty-eight eyes (46 participants) that had Toric IOL implantation, forty-three eyes (31 participants) that had ART +3.0D IOL implantation, were included in the study. All participants were assessed preoperatively, 3, 6, 12 and 24 months postoperatively. Primary outcomes measure: uncorrected distance visual activity (UCDVA), uncorrected near visual acuity (UCNVA), astigmatism and IOL axis alignment, defocus curve, contrast sensitivity and ocular aberration.

Results UCDVA was better than 0.3 LogMAR at 3, 6, 12 and 24 months after surgery in both groups, and UCDVA was significantly improved after surgery than before $(P<0.05)$. All patients with $A R T+3.0 D$ IOL implanted after surgery achieved or exceeded the near-vision of $\mathrm{J} r 3$, which could meet the needs for removal of lens. At $3,6,12$ and 24 months after surgery, the residual astigmatic was $(0.28 \pm 0.20)$ diopters $(D),(0.32 \pm 0.25) D,(0.31 \pm 0.16) D$, $(0.32 \pm 0.22) \mathrm{D}$ in Toric group, and $(0.27 \pm 0.17) \mathrm{D},(0.31 \pm 0.21) \mathrm{D},(0.30 \pm 0.19) \mathrm{D},(0.33 \pm 0.20) \mathrm{D}$ respectively in the ATR group. Compared with preoperative corneal astigmatism, the difference was statistically significant ( $P$ $<0.05)$. The IOL axial mobility of all the patients was less than $5^{\circ}$ in the two groups within 2 years after the operation. There was no difference in aberrations between the ART group and the Toric group under $3 \mathrm{~mm}$ pupil, and there was statistically significant difference between the ART group and the Toric group under $5 \mathrm{~mm}$ pupil ( $P$ $<0.05)$.

Conclusions Toric IOL and ART + 3.OD IOL implantation were safe and effective in the treatment of cataract with corneal astigmatism, and ART + 3.0D IOL provided adequate near vision. The effect had long-term stability.

\section{Background}

With the improvement of cataract surgery technology and the development of IOL, cataract surgery has changed from rehabilitation surgery to refractive surgery. According to statistics, among cataract patients, patients with corneal astigmatism $>1.0 \mathrm{D}$ accounted for $41 \%$, and patients with corneal astigmatism $>1.5 \mathrm{D}$ accounted for $15-$ $29 \%[1,2,3]$. The astigmatism often leads to blurred vision, visual fatigue, eye pain and other discomfort. This study will analyze the long-term clinical effects of Toric IOL and ART +3.0D IOL implantation in the treatment of cataract combined with regular corneal astigmatism.

\section{Methods}

\section{Design of Study Groups}

This study was conducted at Daqing oilfield general hospital, Department of Ophthalmology, and approved by the Institutional Review Board and Ethics Committee of the same hospital (Project No:ZYAF/SC-07/02.0). This study adhered to the tenets of the Declaration of Helsinki and written informed consent was obtained from all patients. Fifty-eight eyes (46 participants) that had Toric IOL implantation, forty-three eyes (31 participants) that had ART +3.0D IOL implantation, were included in the study. All patients were age-related cataract, with regulative corneal morphology and corneal astigmatism $\geq 1.0 \mathrm{D}$. The axis of the eye were between $22.0 \mathrm{~mm}$ and 
$26.0 \mathrm{~mm}$. Patients without any history of lens dislocation, glaucoma, retinal and optic neuropathy, were selected for the study. General conditions of patients are shown in table 1.

\section{table 1. General conditions of patients}

\begin{tabular}{ccccc}
\hline Group & AL[mm $\square$ & Sph[D [ & Cyl(D) & RA(D) \\
\hline Toric group & $23.45 \pm 0.79$ & $22.34 \pm 1.54$ & $1.85 \pm 0.74$ & $0.31 \pm 0.26$ \\
ART group & $23.58 \pm 0.68$ & $22.48 \pm 1.66$ & $1.99 \pm 0.83$ & $0.32 \pm 0.29$ \\
\hline$t$ & 0.581 & 0.563 & 0.984 & 0.438 \\
$P$ & 0.384 & 0.627 & 0.194 & 0.824 \\
\hline
\end{tabular}

$A L=$ Axial length; Sph=Spherical IOL Power; Cyl=Cylindrical IOL Power; $R A=$ Residual astigmatic

\section{Surgical method}

Preoperatively, all patients underwent ophthalmologic examinations, including slit-lamp biomicroscopy, bestcorrected visual acuity (BCVA) measurement, fundus examination, and intraocular pressure measurement. Corneal topogrphy was measured with Pentacam AXL ( Oculus, Wetzlar, Germany). Axial length (AL) and anterior chamber were measured with the IOL-Master (Zeiss 500, Carl Zeiss Meditec, AG). One experienced surgeon performed all surgeries. For preoperative corneal marking, the patient sat up right with the head carefully aligned on the slit lamp. The fine bright slit beam crossed the center of the pupil, and two limbal points (astigmatism axis) of the cornea were marked by dimpling with syringe needle. The surgical procedure was conducted as follows: topical anesthesia, the creation of $2.2 \mathrm{~mm}$ temporal corneal incision, $5.5 \mathrm{~mm}$ continuous curvilinear capsulorhexis, phacoemulsification and aspiration of lens material and IOL implantation using an IOL injector. The patients were implanted with either Toric IOL or ART+3.OD IOL. The same postoperative treatment, consisting of corticosteroids, antibiotics, and nonsteroidal anti-inflammatory eye drops, was administered to all patients.

\section{Postoperative ophthalmic examinations}

The patients were followed up for 2 years. Intraocular pressure, slit-lamp biomicroscopy, UCDVA and IOL axis displacement were examined at 3, 6, 12 and 24 months after surgery in both groups. The patients of ART group examined UCNVA. Defocusing analysis, contrast sensitivity $₫$ OPTEC®6500, STEREO OPTICAL, USA》 and wavefront aberration $₫$ OPD-Scan $\otimes$, NIDEK, CO., LTD, JPN $\otimes$ were detected in the two groups at 2 years after surgery.

\section{Statistical analysis}

Statistical analyses were performed using SPSS ver. 19.0 (SPSS Inc., Chicago, IL, USA). Results were presented as mean, standard deviation. We performed $t$-tests to evaluate the difference between preoperative and postoperative data, and 1-way ANOVA was performed to comparisons of UCDVA at various time. we considered $p \leq 0.05$ statistically significant.

\section{Results}

All 101 eyes (77 patients) were followed up for 2 years, and without secondary glaucoma, intraocular infection, pupil deformation, macular edema and other complications. All patients' IOL were in the capsule and at neutral position. 
Patients in both groups were able to obtain UCDVA better than 0.3 LogMAR at 3, 6, 12 and 24 months after surgery. Postoperative visual acuity was significantly improved compared with that before surgery $(P<0.05)$, as shown in table $2.100 \%$ of IOL patients with implanted ART+3.0D IOL reached or exceeded Jr3 and 91.9\%, 90.8\%, $91.1 \%$ and $87.2 \%$ reached or exceeded $\mathrm{Jr} 1$ at 3, 6, 12 and 24 months after surgery. All patients with ART+3.0D IOL implantation could meet the need for removal of lens after surgery.

\section{Table 2 Preoperative and postoperative UCDVA}

\begin{tabular}{ccc}
\hline Time & Toric group & ART group \\
\hline Preoperative & $1.12 \pm 0.42$ & $1.09 \pm 0.39$ \\
3 months post & $0.08 \pm 0.02^{\mathrm{a}}$ & $0.09 \pm 0.03^{\mathrm{a}}$ \\
6 months post & $0.09 \pm 0.02^{\mathrm{a}}$ & $0.10 \pm 0.02^{\mathrm{a}}$ \\
1 year post & $0.08 \pm 0.04^{\mathrm{a}}$ & $0.10 \pm 0.03^{\mathrm{a}}$ \\
2years post $^{\mathrm{y}}$ & $0.09 \pm 0.03^{\mathrm{a}}$ & $0.10 \pm 0.03^{\mathrm{a}}$ \\
\hline$F$ & 27.46 & 25.81 \\
$P$ & $<0.05$ & $<0.05$ \\
\hline
\end{tabular}

UCDVA=uncorrected distance visual acuity; LogMAR=logarithm of the minimum angle of resolution; ${ }^{a} P<0.05$ versus preoperative uncorrected visual activity.

Astigmatism were $(1.54 \pm 0.64) \mathrm{D},(0.28 \pm 0.20) \mathrm{D},(0.32 \pm 0.25) \mathrm{D},(0.31 \pm 0.16) \mathrm{D},(0.32 \pm 0.22) \mathrm{D}$ in the Toric group at preoperative, $3,6,12$ and 24 months after surgery, and $\varangle 1.61 \pm 0.72 \varangle D,(0.27 \pm 0.17) D,(0.31 \pm 0.21) D,(0.30 \pm 0.19) D$, $(0.33 \pm 0.20) \mathrm{D}$ in ART group. There was no statistically significant difference between the two groups ( $P>0.05)$. There was statistically significant differences between the two groups at each time points after operation and before $(P<0.05)$. There was no statistically significant difference between the residual astigmatism at each postoperative time point and the predicted astigmatism in the two groups ( $P>0.05)$.

$\mathrm{IOL}$ rotation was observed under slit lamp microscope after pupil dilation. $\mathrm{IOL}$ axial rotation was $<5^{\circ}$ in all patients after the operation. The mean absolute IOL rotation of Toric group was $(2.54 \pm 1.31)^{\circ},(2.76 \pm 1.31)^{\circ}$, $(2.91 \pm 1.28)^{\circ},(2.81 \pm 1.30)^{\circ}$ at $3,6,12$ and 24 months after surgery, and the the ATR group was $(2.59 \pm 1.27)^{\circ}$, $(2.84 \pm 1.35)^{\circ},(2.87 \pm 1.31)^{\circ},(2.85 \pm 1.34)^{\circ}$ respectively. There was no statistically significant difference at each time point in the same group $(P>0.05)$.

Figure 1 demonstrates the mean defocus curve of two groups at 24 months after surgery. As shown, visual acuities of ART group better than 0.2 logMAR were observed for defocus levels between +1.00 and $-2.50 \mathrm{D}$, while Toric group were observed between +1.00 and $-1.50 \mathrm{D}$ (Fig. 1).

Figure 1. defocus curve 24 months postoperatively.

Table 3 demonstrates the mean postoperative contrast sensitivity of two groups at 24 months after surgery. The contrast sensitivity of the Toric group at all spatial frequency was slightly better than that in the ART group, regardless of glare or not, but the difference was not statistically significant ( $P>0.05)$.

Table 3. contrast sensitivity at $\mathbf{2 4}$ months after surgery 


\begin{tabular}{ccccccccc}
\hline Groups & \multicolumn{2}{c}{$3 \mathrm{cpd}$} & \multicolumn{2}{c}{$6 \mathrm{cpd}$} & \multicolumn{2}{c}{ 12cpd } & \multicolumn{2}{c}{ 18cpd } \\
\cline { 2 - 9 } & With glare & $\begin{array}{c}\text { Without } \\
\text { glare }\end{array}$ & With glare & $\begin{array}{c}\text { Without } \\
\text { glare }\end{array}$ & With glare & $\begin{array}{c}\text { Without } \\
\text { glare }\end{array}$ & With glare & $\begin{array}{c}\text { Without } \\
\text { glare }\end{array}$ \\
\hline $\begin{array}{c}\text { Toric } \\
\text { group }\end{array}$ & $1.63 \pm 0.24$ & $1.77 \pm 0.16$ & $1.35 \pm 0.17$ & $1.44 \pm 0.14$ & $1.16 \pm 0.23$ & $1.25 \pm 0.22$ & $1.01 \pm 0.25$ & $1.10 \pm 0.10$ \\
ART & $1.45 \pm 0.25$ & $1.64 \pm 0.31$ & $1.24 \pm 0.11$ & $1.29 \pm 0.24$ & $1.05 \pm 0.14$ & $1.19 \pm 0.13$ & $0.92 \pm 0.12$ & $1.01 \pm 0.11$ \\
group & & & & & & & & \\
\hline$t$ & 1.759 & 1.638 & 1.049 & 1.844 & 0.729 & 1.020 & 1.240 & 1.478 \\
$P$ & 0.067 & 0.153 & 0.159 & 0.064 & 0.074 & 0.156 & 0.315 & 0.059 \\
\hline
\end{tabular}

\section{LogMAR=logarithm of the minimum angle of resolution}

Table 4 demonstrates mean postoperative total higher-order aberration data of two groups at 24 monthsafter surgery. There was no statistically significant difference in aberrations at $3 \mathrm{~mm}$ pupil size $(P>0.05)$. The aberrations of the Toric group at $5 \mathrm{~mm}$ pupil size was smaller than that of the ART group, and the difference was statistically significant $(P<0.05)$.

Table 4. total higher-order aberration at 24 months after surgery

\begin{tabular}{ccc}
\hline Group & $3 \mathrm{~mm}(\mu \mathrm{m})$ & $5 \mathrm{~mm}(\mu \mathrm{m})$ \\
\hline Toric group & $0.297 \pm 0.046$ & $0.314 \pm 0.104$ \\
ART group & $0.321 \pm 0.038$ & $0.457 \pm 0.089$ \\
\hline$t$ & 1.557 & 4.228 \\
$P$ & 0.063 & $<0.001$ \\
\hline
\end{tabular}

$3 m m=a b e r r a t i o n s$ at $3 \mathrm{~mm}$ pupil size; $5 \mathrm{~mm}=$ aberrations at $5 \mathrm{~mm}$ pupil size.

\section{Discussion}

Astigmatism is one of the main factors affecting the visual acuity after cataract surgery ${ }^{[4]}$. There are a variety of ways to correct the corneal astigmatism in cataract surgery. For example, corneal incision is made on the steep meridian of corneal curvature. While, even if the application of femtosecond laser assisted corneal incision, there is still a poor predictive, correct the limited scope and back phenomenon, which are susceptible to age, the cornea incision position and size, and corneal thickness. Some studies ${ }^{[5,6,7,8,9,10]}$ have stated that the UCDVA of patients with Toric IOL implantation was better than that of patients with corneal limbal loosening. However, its long-term therapeutic effect has not been reported.

The IOL produced by Alcon company is designed as a sheet of hydrophobic acrylic ester material. The IOL produced on the design platform of Acrysof natural IOL. It have a biconvex 6.0-mm optic, and an overall length of $12.0 \mathrm{~mm}$. The len is available at spherical diopters ranging from +6.0 to $+34.0 \mathrm{D}$ with the cylindrical diopters of Toric IOL ranging from +1.0 to $+6.0 \mathrm{D}$, and the cylindrical diopters of the ART +3.0D IOL rang from + 1.0 to + 3.0 D. Due to their hydrophobic acrylic material material, they have strong adhesion and stability of the bag. What's more, the lens have blue blocking effect, protect the eyes yellow spot and prevent the after cataract ${ }^{[1,12]}[$

The results of this study showed that UCDVA was better than 0.3 LogMAR after cataract phacoemulsification combined with Toric IOL and ART +3.OD IOL implantation within 2 years of follow-up, and significantly improved after surgery than before $(P<0.05)$. After implantation of ART $+3.0 \mathrm{D} \mathrm{IOL}, 100 \%$ of the patients reached or exceeded Jr3 in UCNVA during the 2 years follow-up period, and all of them could meet the requirements of lens 
removal. During the 2 years follow-up period, there was a statistically significant difference in residual astigmatism compared with the preoperative corneal astigmatism $(P<0.05)$, and no statistically significant difference compared with the predicted astigmatism ( $P>0.05)$. The results indicated that Toric IOL and ART+ 3.0D IOL implantation could effectively correct the astigmatism of cataract complicated with good predictability, and the correction effect was stable after 2 years of long-term observation.

In clinical application, only when the axial position of Toric IOL and ART + 3.OD IOL exactly coincide with the meridian of maximum corneal refractive power could the best correction effect be obtained. Novis et al. ${ }^{[13]}$ reported that each rotation of $1^{\circ}$ would lead to the loss of $3.3 \%$ of the lens astigmatism degree, and the correction effect would be reduced by $1 / 3$ if the axial deviation was $10^{\circ}$; deviation of $20^{\circ}$ would reduce the correction effect by about $2 / 3$; Deviation of $30^{\circ}$ increased postoperative astigmatism, and complications such as diplopia, glare and decreased vision would occur. In this study, the axial mobility of IOL in all patients was less than $5^{\circ}$ within 2 years. The results indicated that the IOL position after implantation of Toric IOL and ART+ 3.OD IOL had good long-term stability.

Hoffmann et al. ${ }^{[14]}$ studied patients with AcrySof Toric IOL implantation in 40 eyes, and showed that the average total astigmatism in the whole eye decreased from $(3.55 \pm 0.73) \mathrm{D}$ before surgery to $(0.67 \pm 0.32) \mathrm{D}$ at 3 months after surgery, which is Larger residual astigmatism than observed in this study. Considering the preoperative astigmatism with Hoffmann et al, astigmatism was designed for the whole eye, while our study referred to corneal astigmatism. Visser et al. ${ }^{[15]}$ showed that the UCDVA of 67 eyes was $0.61 \pm 0.26$ after AcrySof Toric IOL T6-T9, and the UCDVA of 70\% eyes was above 0.5 . The rotation degree of IOL 6 months after surgery was (3.2 \pm $2.8)^{\circ}$. The rotation degree of IOL was similar to that of our study, but UCDVA was slightly worse than that of our study, which was considered to be related to the large astigmatism degree observed by Visser. Therefore, accurate preoperative measurement is an important factor to ensure the effect of correction, as well as accurate preoperative marking and intraoperative implantation location. In addition, continuous circular capsulorhexis is very important, and the posterior capsule should be polished, the viscoelastic agent should be completely removed to reduce capsular contraction and epithelial hyperplasia, which is very important to prevent IOL deflection.

The defocus curve can reflect the patient's continuous vision from far to near ${ }^{[16,17]}$. In this study, the longdistance vision of the patients in both groups was close to 0.0 . However, with the increase of the lens power in front of the eyes, the vision of the Toric group decreased rapidly, while the vision of the ART group defocus levels between +1.00 and $-3.00 \mathrm{D}$ could reach more than $0.3 \log$ MAR, displaying $33 \mathrm{~cm}$ from the distance to the eyes, and the patients of the ART group could achieve good vision of more than $0.3 \log M A R$.

Contrast sensitivity is one of the important indicators to reflect visual function. Studies showed that the contrast sensitivity of low spatial frequency was mainly affected by light scattering, while the contrast sensitivity of medium and high spatial frequency was correlated with halo and blurred objects ${ }^{[18,19]}$. As a kind of multi-focal IOL, ART +3.OD IOL inevitably leads to the decline of contrast sensitivity, the decline of visual quality. However, there was no statistical significance difference between single-focal IOL and ART +3.0D IOL in our study $(P>0.05)$.

A large number of literatures ${ }^{[20,21,22]}$ have reported that the decrease of retinal imaging quality after cataract phacoemulsification was closely related to the change of total ocular wavefront aberration. The changes of total 
aberrations of the whole eye after surgery were mainly related to the changes of wavefront aberrations of the

anterior corneal surface and the changes of IOL wavefront aberrations of the replacement ${ }^{[23,24,25,26]}$. The results of this study showed that the total aberration of the whole eye in ART group at $3 \mathrm{~mm}$ pupil was higher than that in the Toric group, but the difference was not statistically significant $(P>0.05)$. Under the condition of $5 \mathrm{~mm}$ pupil, the total aberration of the whole eye in the ART group increased significantly, and the difference was statistically significant compared with that in the Toric group $(P<0.05)$. It suggested that the visual quality of patients in the ART group would decline to some extent when the pupil was large (under dark environment).

When we designed this study, our goal was to evaluate the long-term clinical outcomes of Toric intraocular lens (Toric IOL『and Acrysof IQ Restor Toric +3.0D intraocular lens (ART +3.0D IOL『in patients with cataract and corneal astigmatism. Previous studies were mostly limited to the short-term postoperative period, usually three or six months. This study extended the follow-up period of patients to two years, which more fully demonstrated the long-term effect of astigmatism intraocular lens. In the future, we will continue to follow up the patients in this study to obtain more comprehensive data results.

\section{Conclusion}

Toric IOL and ART +3.OD IOL provided a safe and effective method for treating cataract patients with corneal astigmatism, ART +3.0D IOL could provide patients with adequate close-up vision and improve the dropout rate, without significant decline in visual quality. Dring 2 years of follow-up, this study confirmed that Toric IOL and ART +3.OD IOL implantation had good effects and long-term stability in correcting cataract and corneal regular astigmatism, which has long-term stability.

\section{Abbreviations}

UCDVA: Uncorrected distance visual activity

UCDVA: Uncorrected distance visual activity

UCNVA: Uncorrected near visual acuity

D: Dioptre

AL: Axial length;

Sph: Spherical IOL Power;

RA: Residual astigmatic

\section{Declarations}

Availability of data and materials

The datasets used and/or analysed during the current study are available from the corresponding author on reasonable request. 
Not applicable.

Availibility of data and materials

Not applicable.

Author information

Affiliations

China, Heilongjiang Province, Harbin $₫ 23$ Bundesgasse

the first affiliated hospital of Harbin Medical University

China, Heilongjiang Province, Daqing, 19 ZhongKang Roud

the Daqing Oilfields General Hospital

Contributions

Yang Liu performed the operation of the participants. Ying Su performed ophthalmological evaluation of the participants. Jie Luo and Feng Wang, analyzed and interpreted the data and they are major contributor in writing the manuscript. All authors read and approved the final manuscript.

Corresponding author:

Correspondence to: doctorhrb0451@163.com

Ethics approval and consent to participate

All patients provided written informed consent $\square$ This study adhered to the tenets of the Declaration of Helsinki. This study was approved by Institutional Review Board and Ethics Committee of the Daqing oilfield general hospital (Project no: ZYAF/SC-07/02.0).

Consent for publication

Not applicable.

Competing interests

The authors declare that they have no competing interests.

\section{References}

[1] Fam HB囚Lim KL.Meridional analysis for calculating the expected spherocylindrical refraction in eyes with toric intraocular lenses. Cataract Refract Surg $₫ 2007,33 \llbracket 12 \varangle \llbracket 2072-2076$.

[2] Yoon JJ, Misra SL, McGhee CN, Patel DV. Demographics and ocular biometric characteristics of patients undergoing cataract surgery in Auckland, New Zealand. Clin Exp Ophthalmol 2016;44:106-13. 
[3] Pan CW, Cheng CY, Sabanayagam C, et al. Ethnic variation in central corneal refractive power and steep cornea in Asians. Ophthalmic Epidemiol 2014;21:99-105.

[4] Kim MJ, Yoo YS, Joo CK, Yoon G. Evaluation of optical performance of 4 aspheric toric intraocular lenses using an optical bench system: Influence of pupil size, decentration, and rotation. Cataract Refract Surg 2015;41:2274-82.

[5] Mendicute J, Irigoyen C, Ruiz M, et al.Toric intraocular lens versus opposite clear corneal incisions to correct astigmatism in eyes having cataract surgery. Cataract Refract Surg, 2009, 35(3):451-458.

[6] Kessel L, Andresen J, Tendal B, et al. Toric intraocular lenses in the correction of astigmatism during cataract surgery: a systematic review and meta-analysis. Ophthalmology 2016;123:275-86.

[7] Zhu X, He W, Zhang K, Lu Y. Factors influencing 1-year rotational stability of AcrySof Toric intraocular lenses. Ophthalmol 2016;100:263-8.

[ 8] Kretz FT, Breyer D, Klabe K, et al. Clinical outcomes and capsular bag stability of a four-point haptic bitoric intraocular lens. Refract Surg 2015;31:431-6.

[9] Mencucci R, Favuzza E, Guerra F, et al. Clinical outcomes and rotational stability of a 4-haptic toric intraocular lens in myopic eyes. Cataract Refract Surg 2014;40:1479-87.

[10] Ouchi M. High-cylinder toric intraocular lens implantation versus combined surgery of low-cylinder intraocular lens implantation and limbal relaxing incision for high-astigmatism eyes. Clin Ophthalmol 2014;8:661-7.

[11] Werner L, Olson RJ, Mamalis N, et al. New technology IOL optics. Ophthalmol Clin North Am, 2006, 19(4):469-483.

[12] Kwartz J, Edwards K. Evaluation of the long-term rotational stability of single-piece, acrylic intraocular lenses. Br J Oph-thalmol, 2010, 94(8):1003-1006.

[13] Novis C. Astigmatism and toric intraocular lenses. Current Opinion in Ophthalmology, 2000, 11(1):47-50.

[14] Hoffmann PC, Auel S, Hũtz WW. Results of higher power toric intraocular lens implantation. Cataract Refract Surg, 2011, 37(8):1411-1418.

[15] Visser N, Ruíz-Mesa R, Pastor F, et al. Cataract surgery with toric intraocular lens implantation in patients with high cormeal astigmatism. Cataract Refract Surg, 2011, 37(8):1403-1410.

[16] ALI J L, KAYMAK H, BREYERD, COCHENER B, PLAZAPUCHE A B. Quality of life related variables measured for three multifocal diffractive intraocular lenses: a prospective randomised clinical trial. Clin Exp Ophthalmol, 2017, 16(10):1442-9071》

[17] MCNEELY R N, PAZO E, SPENCE A, RICHOZ O, NESBIT M A, MOORE T C B, et al. Visual outcomes and patient satisfaction 3 and 12 months after implantation of a refractive rotationally asymmetric multifocal intraocular lens. Cataract Refract Surg, 2017, 43(5):633-638》 
[18] MANTES-MICO R, ALIO JL. Distance and near contrast sensitivity function after multifoeal intraecular lenses implantation. Cataract Refract Surg, 2003, 29(4): 703.

[19] ZHAO YE, ZHANG GL, WANG QM, et al. Observation of multifocal intraocular lens implantation of visual function. Chinese Journal of Ophthalmology, 2005, 41(4): 369.

[20] Kamiya K, Shimizu K, Miyake T. Changes in astigmatism and corneal higher-order aberrations after phacoemulsification with toric intraocular lens implantation for mild keratoconus with cataract. Jpn J Ophthalmol 2016;60:302-8.

[21] Chang DH囚Rocha KM. Intraocular lens optics and aberra-tions. Curr Opin Ophthalmol囚2016,27(4): 298-303囚

[22] Liao X, Lin J, Tian J, et al. Evaluation of optical quality: ocular scattering and aberrations in eyes implanted with diffractive multifocal or monofocal intraocular lenses. Curr Eye Res $₫ 2018$, 43(6): 696-701囚

[23] IseliHP, JankovM,BueelerM,etal.Corneal and total wavefront aberrations in phakic and pseudophakic eyes after implantation of monofocal foldable intraocular lenses. Cataract Refract Surg,2006,32(5):762-771.

[24] Guirao A,Tejedor J,Artal P.Corneal aberrations before and after small-Incision cataract surgery. Invest Ophthalmol Vis Sci,2004,45(12):4312 -4319.

[25] Artal P,Guirao A.Contributions of the cornea and the lens to the aberrations of the human eye.Opt Lett,1998,23(9):1713-1715.

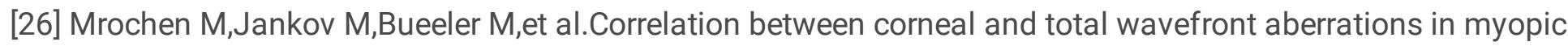
eyes.Refract Surg,2003,19(2):104-112.

\section{Figures}




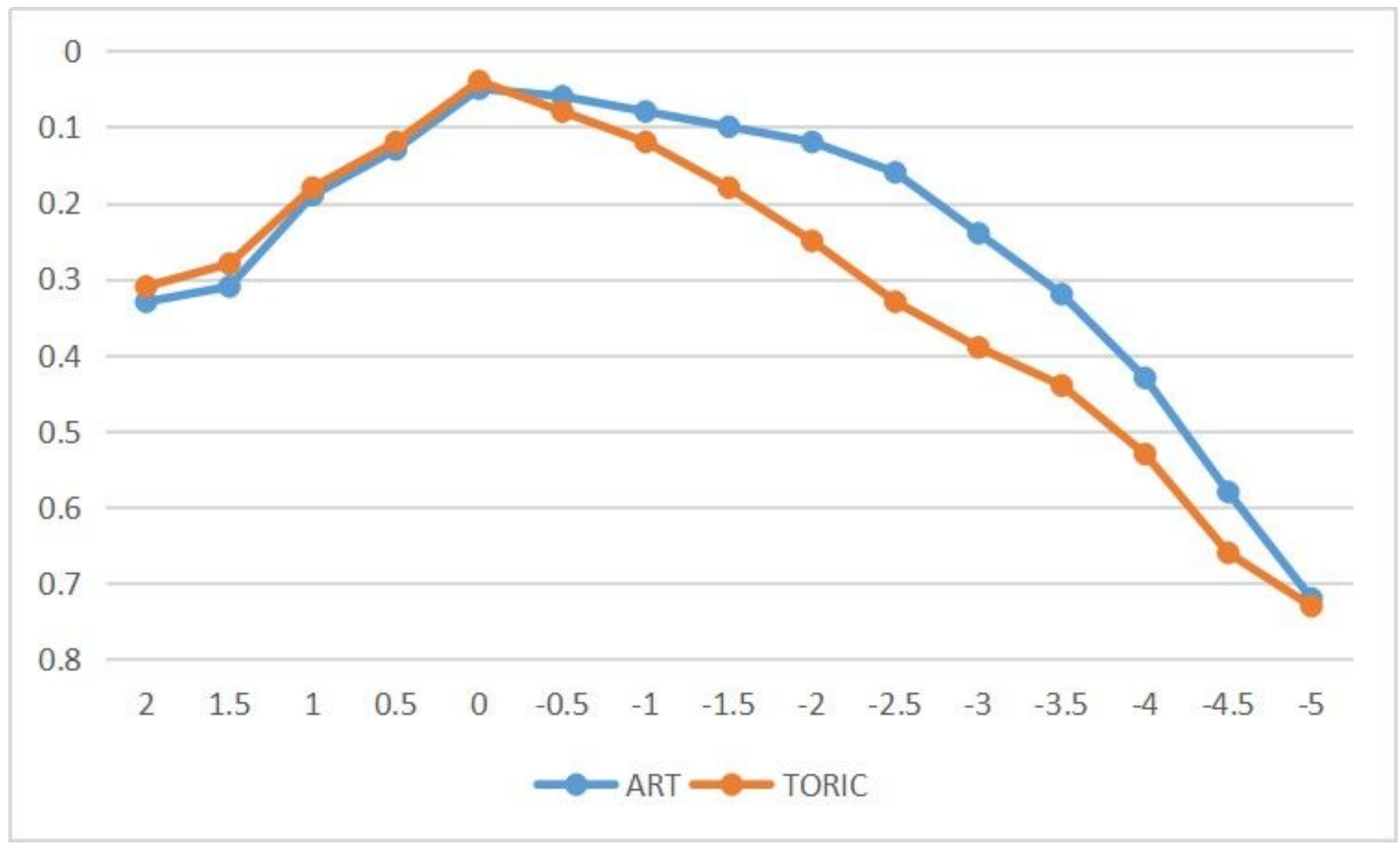

Figure 1

defocus curve 24 months postoperatively. 